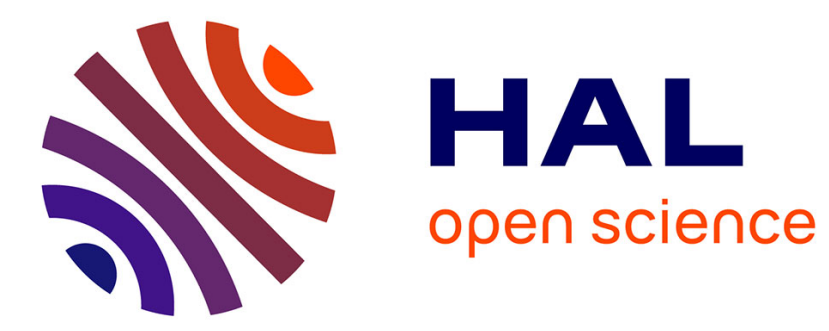

\title{
Structural, vibrational, and magnetic properties of self-assembled CoPt nanoalloys embedded in SrTiO 3
}

M. Hennes, E. Fonda, N. Casaretto, J. Buchwald, X. Weng, G. Patriarche, D. Demaille, Y. Zheng, F. Vidal

\section{- To cite this version:}

M. Hennes, E. Fonda, N. Casaretto, J. Buchwald, X. Weng, et al.. Structural, vibrational, and magnetic properties of self-assembled CoPt nanoalloys embedded in SrTiO 3. Physical Review Materials, 2020, 4 (12), 10.1103/PhysRevMaterials.4.126001 . hal-03095549

\section{HAL Id: hal-03095549 \\ https://hal.science/hal-03095549}

Submitted on 4 Jan 2021

HAL is a multi-disciplinary open access archive for the deposit and dissemination of scientific research documents, whether they are published or not. The documents may come from teaching and research institutions in France or abroad, or from public or private research centers.
L'archive ouverte pluridisciplinaire $\mathbf{H A L}$, est destinée au dépôt et à la diffusion de documents scientifiques de niveau recherche, publiés ou non, émanant des établissements d'enseignement et de recherche français ou étrangers, des laboratoires publics ou privés. 


\title{
Structural, vibrational, and magnetic properties of self-assembled CoPt nanoalloys embedded in $\mathrm{SrTiO}_{3}$
}

\author{
M. Hennes, ${ }^{1, *}$ E. Fonda, ${ }^{2}$ N. Casaretto, ${ }^{1}$ J. Buchwald,${ }^{3}$ X. \\ Weng, ${ }^{1}$ G. Patriarche, ${ }^{4}$ D. Demaille, ${ }^{1}$ Y. Zheng, ${ }^{1}$ and F. Vidal ${ }^{1, \dagger}$ \\ ${ }^{1}$ Sorbonne Université, CNRS, Institut des NanoSciences de Paris, INSP, F-75005 Paris, France ${ }^{\ddagger}$ \\ ${ }^{2}$ Synchrotron Soleil, L'Orme des Merisiers Saint-Aubin BP 48, 91192 Gif-sur-Yvette Cedex, France \\ ${ }^{3}$ Institute for Materials Science and Max Bergmann Center of Biomaterials, \\ Dresden University of Technology, 01062 Dresden, Germany \\ ${ }^{4}$ Centre de Nanosciences et de Nanotechnologies (C2N), \\ CNRS, Université Paris Sud, Université Paris Saclay
}

(Dated: April 28, 2020)

\begin{abstract}
Pulsed laser deposition was used to synthesize vertically aligned nanocomposites (VAN), consisting of ultrathin CoPt nanocolumns embedded in a single crystal $\mathrm{SrTiO}_{3}$ thin film. Combining highresolution transmission electron microscopy (HRTEM), x-ray diffraction (XRD), x-ray absorption spectroscopy (XAS) and molecular dynamics (MD) simulations, we provide an in-depth analysis of the structural and vibrational properties of the CoPt phase over a wide range of concentrations. Despite growing the samples at high temperatures $\left(>600^{\circ} \mathrm{C}\right)$, we find that the CoPt nanoalloys are characterized by a high degree of structural disorder and complete absence of chemical ordering, even after additional sample annealing. Unexpectedly, for a Co concentration exceeding $c(\mathrm{Co}) \simeq 0.55$, pole figure measurements unravel a transition from a highly textured short range disordered fcc phase to a disordered hcp phase with c-axis oriented out of plane. These findings, further confirmed by magnetometry measurements, illustrate the profound impact of vertical epitaxy on the structural and thermodynamic properties of bimetallic CoPt alloys.
\end{abstract}

\section{INTRODUCTION}

During the last two decades, considerable research effort has been devoted to the development of functional oxide thin films with embedded metallic nanostructures. A broad variety of synthesis techniques has been proposed to grow these hybrid metal-oxide systems: ion implantation ${ }^{1}$, low energy cluster beam deposition assisted by co-deposition of the matrix ${ }^{2-4}$, ion-irradiation induced viscous flow ${ }^{5-8}$, laser treatment ${ }^{9}$ or electrodeposition into prestructured templates ${ }^{10,11}$ have been studied in great detail. However, these approaches still lack the required versatility when one is interested in controlling the nanoarchitecture of the composite system, i.e., the morphology and crystallographic orientation of the embedded metallic structure.

Vertical epitaxy, the three-dimensional counterpart of classical planar epitaxy, has recently emerged as a novel field of research, with the potential ability to fill this gap. In fact, it has been demonstrated that in mixed metaloxide systems, nanoscale self-assembly driven by phase separation yields composites with highly tunable crystallographic properties. In addition, careful adjustment of the growth conditions, i.e. deposition rate, temperature or concentration can be used to achieve precise control over the aspect ratio and morphology of the metallic inclusions ${ }^{12}$. As shown in recent studies, the aforementioned approach seems applicable to a wide range of materials: successful synthesis of epitaxial $\mathrm{Fe}^{13}, \mathrm{Co}^{14-17}$, $\mathrm{Ni}^{12,18-20}, \mathrm{Au}^{21}, \mathrm{Ir}^{22}, \mathrm{Pt}, \mathrm{Pd}$ and $\mathrm{Rh}^{23}$ nanowire and nanopillar arrays, embedded in different types of oxide matrices has been reported. However, only few experiments on alloy-based composites have been performed yet $^{24,25}$. Within this context, CoPt is a particularly interesting candidate from a fundamental, as well as from a technological point of view. On the one hand, it is an archetypal alloy, which has been studied in detail for its disorder-order phase transitions ${ }^{26}$. On the other hand, some of the ordered phases display a huge uniaxial magnetic anisotropy, which makes them appealing for high density data storage and spintronics applications. Usually, CoPt thin films are grown at room temperature and require subsequent annealing steps to achieve chemical ordering ${ }^{27,28}$. In contrast, the synthesis of vertically aligned metal-oxide nanowire composites must be performed at high temperatures $\left(T>600^{\circ} \mathrm{C}\right)$ to activate surface diffusion and achieve substantial phase separation $^{12}$. While this might concomitantly pave the way for the creation of ordered bimetallic phases during growth, it is also well known that chemical ordering can be impeded due to size and shape effects ${ }^{29}$. Matrix atom diffusion into the embedded metals and compound formation can also play an important role and have a detrimental impact on the magnetic properties of $\mathrm{CoPt}^{30,31}$.

In the present work, we use $\mathrm{CoPt}-\mathrm{SrTiO}_{3}$ as a model system to study the interplay of these effects and analyze whether or not, chemical ordering takes places when ultrasmall nanoalloys self-assemble in a single crystal matrix. Several studies have focused on deposition of CoPt alloy thin films and nanostructures using single crystal $\mathrm{SrTiO}_{3}$ substrates ${ }^{32-34}$. However, to the best of our knowledge, no data is available yet concerning the possibility to grow metal-oxide composite structures using these materials. In the first part of this paper, we demonstrate that co-deposition of $\mathrm{Co}, \mathrm{Pt}$ and $\mathrm{SrTiO}_{3}$ at high temperatures indeed results in self-assembly of 
(a)

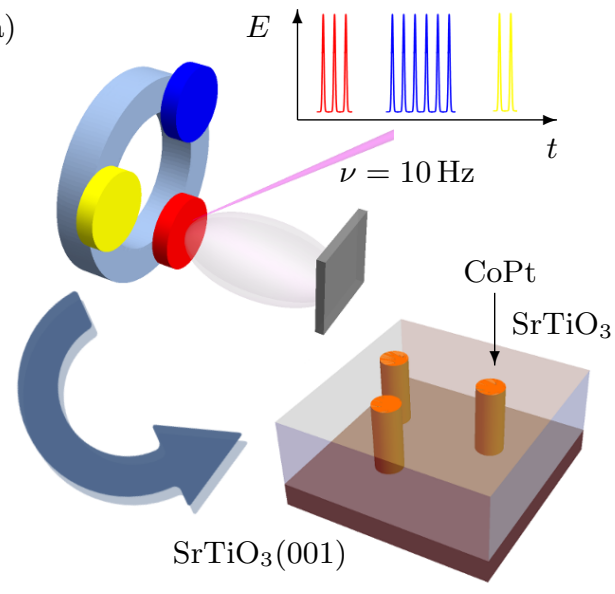

(b)

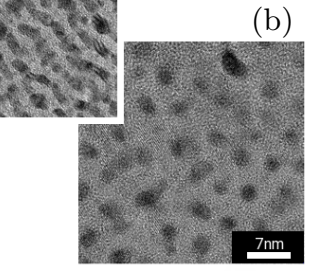

(c)

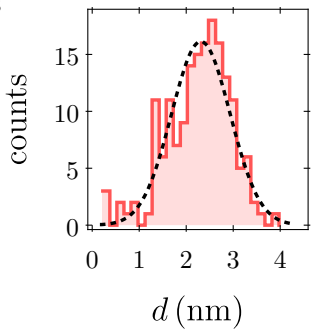

(d)

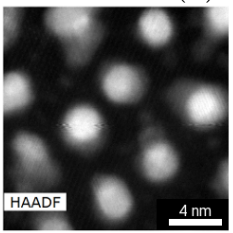

(e)

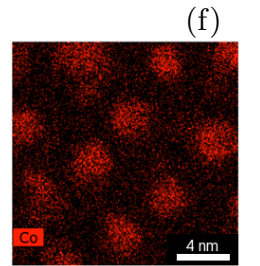

$(\mathrm{g})$

(h)

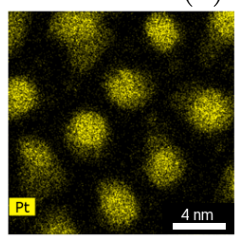

(i)

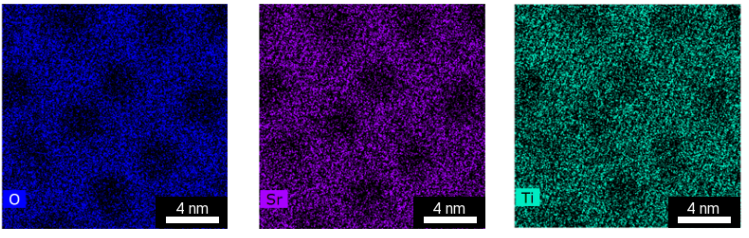

FIG. 1. Schematics of the growth procedure and exemplary electron microscopy data. (a) Sequential pulsed laser deposition using $\mathrm{Pt}, \mathrm{CoO}$ and $\mathrm{SrTiO}_{3}$ targets, results in growth of $\mathrm{CoPt}-\mathrm{SrTiO}_{3}$ nanocomposites. (b) HR-TEM plan view and tilted view, which unravels the columnar morphology of the nanoinclusions in the single crystal $\mathrm{SrTiO}_{3}$ matrix. (c) Diameter distribution of the embedded structures (sample S4) (d-i) plan view STEM-HAADF and EDX data (sample S1) provide detailed chemical information on the nanocomposite and confirm phase separation and creation of CoPt nanoalloys.

nanocolumnar metal-oxide thin films. We then turn to an in-depth description of their peculiar structural and magnetic properties before closing with a conclusion and outlook.

\section{B. Structural and magnetic measurements}

The structure of the samples was studied using a combination of techniques including transmission electron microscopy (TEM) and scanning transmission electron microscopy (STEM), x-ray diffraction (XRD), and x-ray absorption spectroscopy (XAS). High resolution transmission electron microscopy (HRTEM) data were acquired using a JEOL JEM 2100F equipped with a fieldemission gun operated at $200 \mathrm{kV}$. STEM data were gathered using a Titan Themis 200. The surface structure of the samples was analyzed using a Zeiss Ultra55 scanning electron microscope (SEM). Energy-dispersive Xray spectroscopy (EDX) was used to map the chemical distribution of the elements using all three electron microscopes mentionned above. X-ray diffraction (XRD) data were obtained on a laboratory diffractometer (Rigaku SmartLab) with $\mathrm{Cu} \mathrm{K}_{\alpha 1}$ radiation (wavelength of $1.54 \AA$ ), reflectivity measurements (XRR) were used to determine the thickness of the thin films. Co K edge and $\mathrm{Pt} \mathrm{L}_{3}$ edge X-ray absorption spectroscopy (XAS) data were collected at the beamline SAMBA (Synchrotron SOLEIL). All samples were probed in fluorescence detection mode. A commercial VSM system was used to record temperature-dependent magnetization curves (PPMS, Quantum Design) in in-plane and out-of-plane geometry. 
TABLE I. Structural parameters and composition of the $\mathrm{Co}_{x} \mathrm{Pt}_{1-x}$-STO samples. Average nanorod diameters $\langle D\rangle$ and porosities $p$ determined from plan-view TEM micrographs, sample thickness $t$ obtained via XRR. Concentrations measured via SEM-EDX $\left(c_{\mathrm{I}}\right)$, HRTEM-EDX $\left(c_{\mathrm{II}}\right)$ and STEM$\operatorname{EDX}\left(c_{\text {III }}\right)$.

\begin{tabular}{lcccccc}
\hline \hline & & \multicolumn{5}{c}{ Co concentration } \\
Sample & $\langle D\rangle(\mathrm{nm})$ & $p(\%)$ & $t(\mathrm{~nm})$ & $c_{\mathrm{I}}(\mathrm{at} \%)$ & $c_{\text {II }}(\mathrm{at} \%)$ & $c_{\text {III }}(\mathrm{at} \%)$ \\
\hline S0 & $2.5(5)$ & 16 & 73 & 0 & 0 & - \\
S1 & $2.5(4)$ & 12 & 66 & $20(2)$ & 25 & $15(5)$ \\
S2 & $3.5(9)$ & 21 & 57 & $42(4)$ & 42 & $32(4)$ \\
S3 & $2.6(6)$ & 17 & 76 & $51(2)$ & 52 & - \\
S4 & $2.7(7)$ & 19 & 44 & $57(5)$ & 57 & - \\
S5 & $2.4(8)$ & 17 & 73 & $57(3)$ & 63 & $48(3)$ \\
S6 & $2.6(6)$ & 20 & 57 & $67(2)$ & 75 & - \\
S7 & $1.8(8)$ & 13 & 32 & 100 & 100 & - \\
\hline \hline
\end{tabular}

\section{MEAM-based MD simulations}

Classical molecular simulations were carried out with the LAMMPS $^{35}$ code using a cell containing 2916 atoms and periodic boundary conditions. The interactions between the atoms were described in terms of the second nearest-neighbor modified embedded atom method $(\mathrm{MEAM})^{36}$ using the parameterization of Kim et $a l .{ }^{37}$. After 10 ps equilibration of the cell to a mean pressure of 0 bar within the NPT ensemble, a run was conducted within the canonical ensemble at $300 \mathrm{~K}$ using NoséHoover thermostating ${ }^{38,39}$. All runs were performed over a time span of $100 \mathrm{ps}$ with a time step of $1 \mathrm{fs}$.

\section{RESULTS AND DISCUSSION}

\section{A. Bimetallic nanopillar formation}

We start this section by providing evidence for phase separation and nanopillar formation in the CoPt-STO thin films. Fig. 1(b) shows a representative HR-TEM image of sample $\mathrm{S} 4$. In this plan view, nanoinclusions with irregular but mostly disk-like shape can easily be identified. Tilting of the sample $\left(\theta=25^{\circ}\right)$ revealed the columnar morphology of the nanocomposite (inset of Fig 1(b)), which has been found in all samples, irrespective of concentration. The densities and diameters of the embedded structures were quantified using Mathematica ${ }^{\circledR}$-based image processing scripts, which permitted to analyse a large number of plan view micrographs (a detailed summary of the results obtained for various Co and Pt concentrations is given in Table I). The average diameters of the columns were found to lie roughly between $2 \mathrm{~nm}$ and $3.5 \mathrm{~nm}$, with no systematic change upon variation of the concentration. Fig 1(c) shows an exemplary diameter distribution obtained on sample S4. While the latter exhibits an overall Gaussian shape, slight devia- tions become apparent for small diameters. Indeed, in all samples, a second peak, typically below $d \simeq 1.5 \mathrm{~nm}$, is observed, hinting at a small amount of additional buried structures. This is confirmed by careful analysis of STEM-HAADF maps, which provide better contrast due to high $\mathrm{Z}$ sensitivity. As shown in Fig1(d), small clusters with faint contrast located inbetween the pillars can be identified.

To complement our morphological analysis, quantitative EDX data of the thin films were acquired with different spatial resolution, giving access to chemical information on various length scales (Table I). Low resolution maps, were obtained by using SEM-EDX and scanning point arrays over the entire sample to improve the statistics. EDX data gathered with this approach unraveled the lateral homogeneity of the thin films. This was further confirmed by using HRTEM-EDX. Data obtained when scrutinizing thin film regions containing several thousand nanowires (typical length scales close to $100 \mathrm{~nm}$ ) were found in very good agreement with the aforementioned results. In addition, samples S1, S2 and S5 were analyzed in greater detail using STEM-HAADF and STEM-EDX, providing chemical information down to sub-nm length scales. As exemplified in Fig.1(e-i), the columnar inclusions display strong $\mathrm{Co}$ and $\mathrm{Pt}$ signals, correlating with low $\mathrm{Sr}, \mathrm{Ti}$ and $\mathrm{O}$ intensities. This provides strong evidence for phase separation and creation of CoPt alloy nanostructures. Surprisingly, STEMbased quantitative chemical analysis of embedded metallic CoPt yielded systematic concentration deviations of roughly 10-15\%, when compared to the low resolution EDX data: all samples appear to be slightly Co deficient (Table I). This holds true for isolated CoPt columns as well as for larger matrix-nanowire composite regions (probed lateral length scales of approximately $10 \mathrm{~nm}$ ), which display almost identical concentrations. Thus, the observed discrepancy cannot be attributed to cobalt enrichment in the surrounding STO.To resolve this apparent contradiction, it is necessary to recall that STEMEDX mapping not only yields a highly local chemical analysis of the sample in plane, its vertical sensitivity also differs from the two aforementioned EDX techniques. In fact, only the upper $10-15 \mathrm{~nm}$ of the thinned film were probed in order to achieve ideal electron beam transparency. In contrast, the SEM-EDX and HRTEM-EDX analysis presented above allowed to assess the chemical composition of the composite thin film from its surface down to the substrate interface. The observed differences thus hint at a weak concentration gradient in the thin films, which appear to be slightly Co enriched at the substrate interface, while presenting a larger Pt concentration at their surface. Finally, quantitative chemical analysis of the matrix indicates that, within measurement uncertainty, the concentration of $\mathrm{Ti}, c(\mathrm{Ti})$, approximately equals the concentration of $\mathrm{Sr}, c(\mathrm{Sr})$ and we obtain $c(\mathrm{Ti}) \simeq c(\mathrm{Sr}) \simeq 1 / 3 \cdot c(\mathrm{O})$. This is in agreement with the expected formation of $\mathrm{SrTiO}_{3}$ and further confirmed by our TEM data as well as XRD measurements, that 

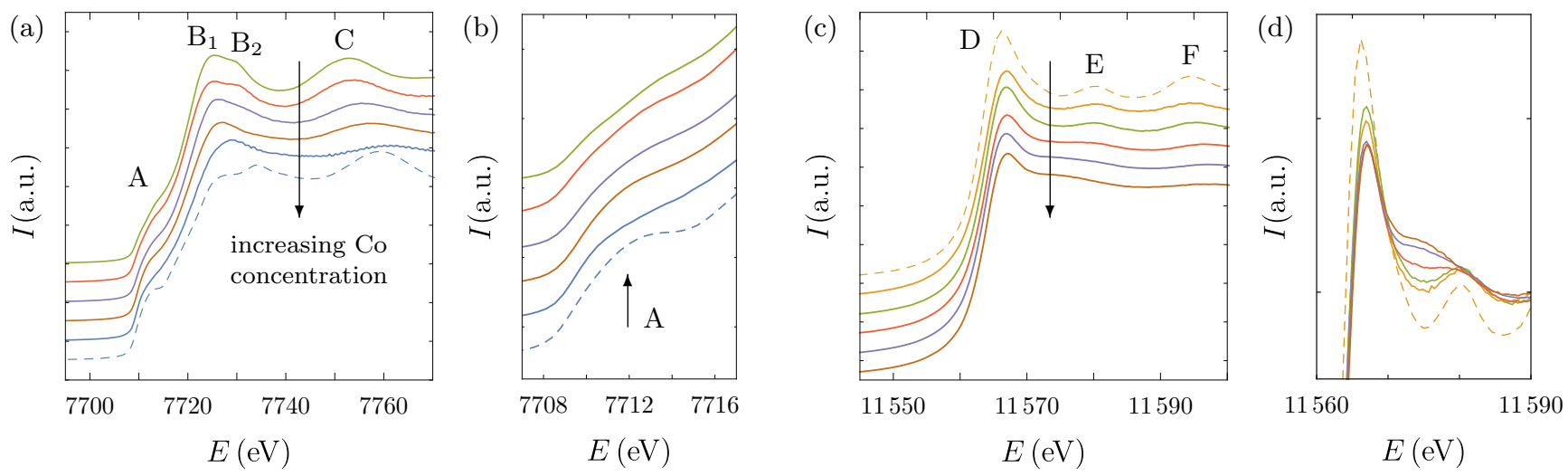

FIG. 2. XANES data obtained at the Co K- and $\mathrm{Pt} \mathrm{L}_{3}$-edges (increasing Co concentration from top to bottom) and main features labeled from A to F: Co and Pt foil (dashed blue and dashed orange, respectively) and samples S7 (blue), S6 (brown), S4 (purple), S2 (red), S1 (green) and S0 (orange). (a) Co K-edge, (b) magnified spectra illustrating the decreasing magnitude of peak A with increasing Pt concentration. (c) Pt $\mathrm{L}_{3}$-edge and (d) zoom on the white line. Note that the curves in (a-c) were shifted vertically for clarity.

will be presented in a later part of this work. Note that the presence of minute amounts of $\mathrm{Sr}$, $\mathrm{Ti}$ and $\mathrm{O}$ on top of $\mathrm{Co}$ and $\mathrm{Pt}$ in plan view, as seen in $1(\mathrm{~d})$, results from the thin $\mathrm{SrTiO}_{3}$ capping layer, used to prevent oxidation and contamination.

\section{B. Alloying and absence of chemical short range ordering}

While the combined SEM, TEM and EDX analysis, presented in the last section, allows to conclude that shape anisotropic CoPt-rich inclusions are formed inside the STO matrix during growth, they provide little information on a possible oxide or compound creation in the matrix/nanorod interface region. In addition, EDX mapping does not allow to discriminate between chemically ordered and disordered phases. To complement our structural analysis, we therefore performed x-ray absorption spectroscopy measurements which will be presented in the following paragraphs.

\section{X-ray absorption spectra (XAS) of as-deposited samples}

Figure 2 shows x-ray absorption near edge spectra (XANES) recorded at the Co K-edge and $\mathrm{Pt} \mathrm{L}_{3}$-edge for nanocomposites with varying $\mathrm{Co}$ and $\mathrm{Pt}$ concentration, as well as reference spectra measured on $\mathrm{Co}$ and $\mathrm{Pt}$ foils. At the Co K-edge, 3 distinct features (labeled A, B and C) can be identified in the near-edge region (Fig. 2(a)). Peak A reflects transitions from 1s core levels to unoccupied states exhibting mainly $3 \mathrm{~d}$ character while the peak B results from final configurations with $4 \mathrm{p}$ character ${ }^{40-43}$. Previous work has evidenced that the magnitude of these local maxima in CoPt alloys depends on the concentra- tion, reflecting their sensitivity upon modifications in the alloy electronic density of states. As can clearly be infered from Fig. 2b, the magnitude of peak A decreases with increasing $\mathrm{Pt}$ concentration in the samples. This behavior agrees with data reported in earlier studies ${ }^{40,41,44}$ and has been explained theoretically by an increase in $3 \mathrm{~d}$ electron occupancy at Co sites when increasing the $\mathrm{Pt}$ concentration. In contrast, the feature B is less straightforward to analyze in the present case. While a reduction in peak intensity with increasing Co concentration was demonstrated (and traced back to a reduction of $4 \mathrm{p}$ states resulting from an alloying induced weakening of p-d hybridization at Co sites ${ }^{41}$ ), our data reveal a concomittant change in peak shape with concentration. Indeed, for high values of $c(\mathrm{Pt}), \mathrm{B}$ is composed of two maxima $B_{1}$ and $B_{2}$, which arise from band structure effects. These separated structures merge into a single peak with increasing $c(\mathrm{Co})$, which suggests a loss of crystallinity in the NWs with higher Co concentration ${ }^{20}$. Finally, peak $\mathrm{C}$ can be attributed to fine structure oscillations: with increasing Pt content, a shift towards lower energies (larger interatomic distances) is observed. A detailed analysis of the extented x-ray absorption fine structure (EXAFS) part of the spectrum will be given in the next section. At the $\mathrm{Pt} \mathrm{L}_{3}$ edge, differences between the spectra are less pronounced (Fig.2c). The most prominent feature in the Pt XANES is the white line, reflecting $2 p_{3 / 2} \rightarrow 5 d_{5 / 2}$ transitions ${ }^{45}$ (peak D in Fig. 2c). As shown in Fig. 2d, superposition of the spectra reveals a decrease of the white line intensity and broadening with increasing Co concentration. This effect is rather small and saturates around $40 \%$ Co concentration, in agreement with literature data ${ }^{41}$.

While XANES data confirm phase separation and growth of metallic CoPt structures embedded in $\mathrm{SrTiO}_{3}$, they do not provide details on the degree of ordering of 

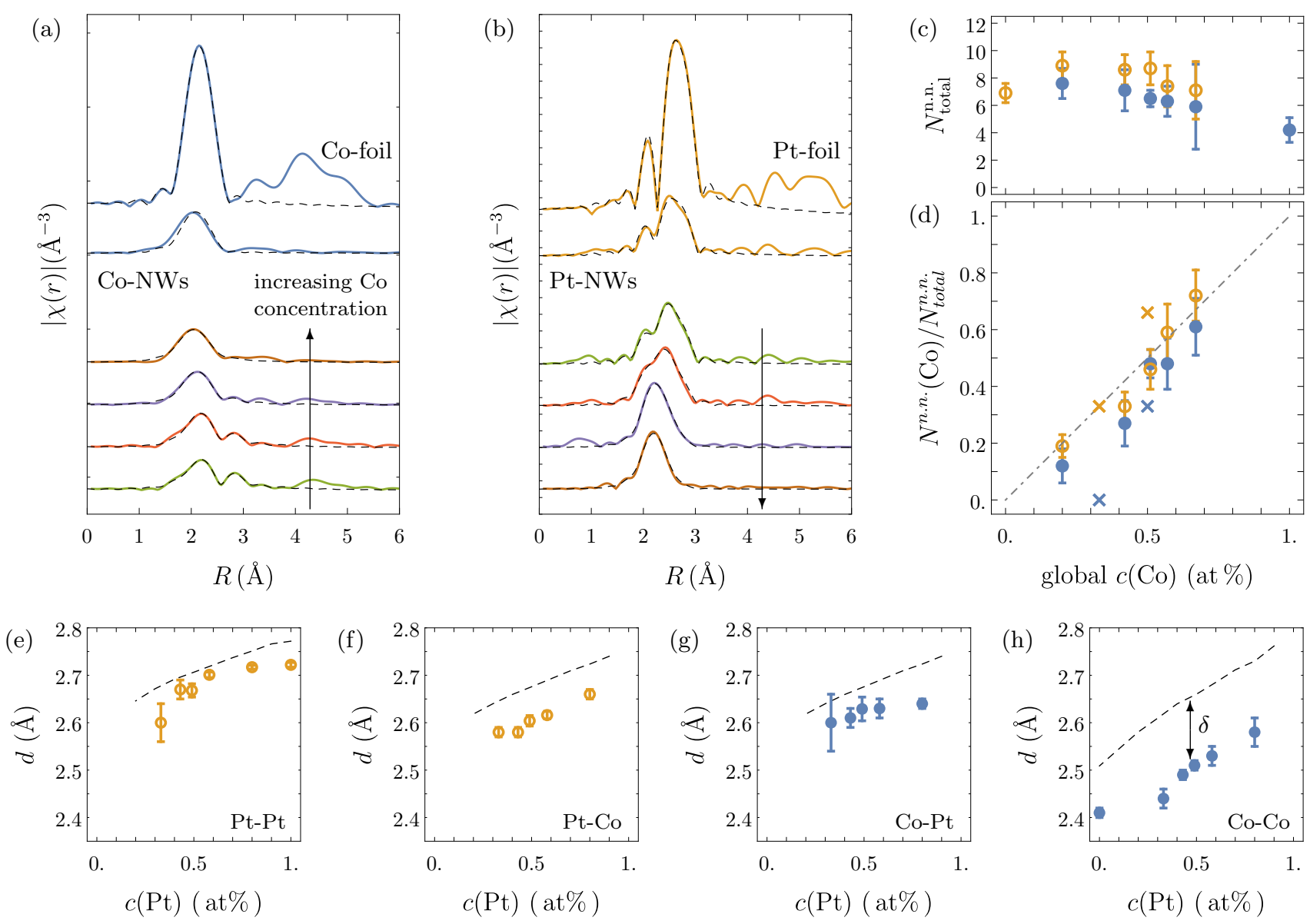

FIG. 3. EXAFS measured on nanocolumnar $\mathrm{CoPt}-\mathrm{SrTiO}_{3}$ samples with varying $\mathrm{Co} / \mathrm{Pt}$ ratio (arrows indicate increasing Co concentrations). Co and Pt foil data are shown for comparison. $\chi(r)$ data obtained after Fourier transformation of $k^{2} \chi(k)$ at the (a) Co K-edge and (b) Pt L ${ }_{3}$-edge (same color coding as in Fig.2) as well as corresponding fits (black dashed line). (c) Total number of next neighbors (n.n.) as a function of concentration, (d) local Co concentration vs global Co concentration (crosses indicate the values expected for pure $\mathrm{L} 1_{0}$ and $\mathrm{L}_{2}$ phases), n.n. distances for (e) Pt-Pt, (f) Pt-Co, (g) Co-Pt and (h) Co-Co bonds (blue solid points: Co K-edge, orange circles: Pt L ${ }_{3}$-edge, black dashed line: MEAM results).

the alloy ${ }^{46}$. In contrast, analysis of the fine structure permits to gather information on the type and the number of neighboring atoms, as well as on distances separating the absorber and the scatterer ${ }^{47}$. For data analysis, the IFEFFIT libraries were used $^{48}$. Raw spectra were treated with ATHENA to remove the background and glitches and to normalize the data. ARTEMIS was then used for quantitative EXAFS fitting. To achieve maximum consistency, an identical analysis procedure was applied to all samples. Measurements on Co and Pt foils were first used to calculate the amplitude reduction factor $S_{0}^{2}$ $\left(N=12, S_{0}(\mathrm{Co})=0.8 \pm 0.02\right.$ and $\left.S_{0}(\mathrm{Pt})=0.86 \pm 0.03\right)$. We then fitted all nanocomposite spectra by assuming Co and $\mathrm{Pt}$ atoms to be exclusively surrounded by either $\mathrm{Co}$ or Pt neighbors. In these calculations, only next neighbors (n.n.), i.e., the first coordination shell was taken into consideration. Fourier transformations of $\chi(k)$ spectra were performed in the range $3 \AA^{-1}<k<11 \AA^{-1}$ using a Hanning window with $d k=1 \AA^{-1}$. Six model- ing parameters per edge were used: an energy correction factor $\Delta E$, two n.n. distances $d$ (one for each type of neighbor), two total n.n. numbers $N\left(N_{\mathrm{Co}}\right.$ and $\left.N_{\mathrm{Pt}}\right)$ and one Debye-Waller factor $\sigma$. While, for the present system, it would seem advisable to choose different disorder parameters to describe Co-Pt and Co-Co as well as PtCo and Pt-Pt bonds, this approach did not result in a consistent fitting of the data and produced large errors. We therefore sticked to a single EXAFS Debye-Waller parameter $\sigma$ per edge in the first place. Similarly, we found that a third cumulant $C_{3}$, usually employed in nanoscale systems as a correction for non-Gaussian distance distributions, worsened the fits and no clear evolution of $C_{3}$ with concentration could be identified.

Figures $3(\mathrm{a}-\mathrm{b})$ show $\chi(r)$ data obtained by Fourier transformation of $k^{2}$ weighted $\chi(k)$ spectra gained on $\mathrm{CoPt}$ alloy composites together with corresponding fits. $\mathrm{Co}$ and $\mathrm{Pt}$ foils and elemental nanocomposites are presented as well. In general, the agreement between the 
(a)

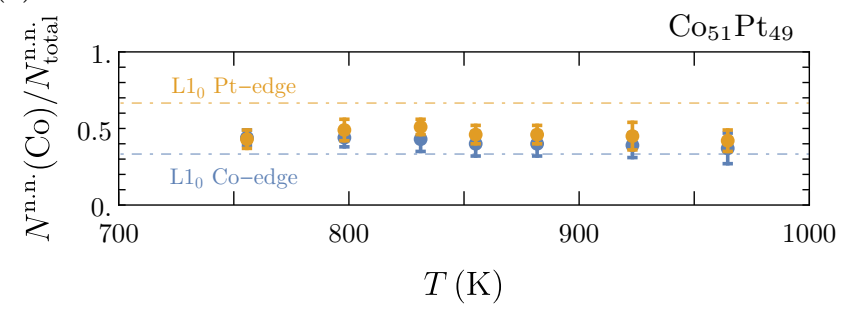

(b)
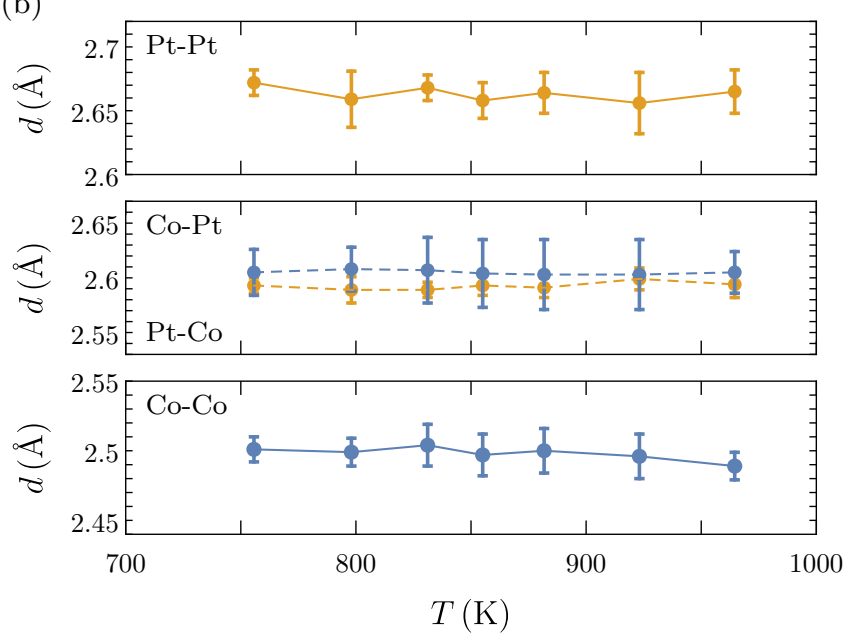

FIG. 4. In situ annealing study performed on sample S3, using annealing times $t_{a}^{\star}=10 \mathrm{~h}($ at $750 \mathrm{~K})$ and $t_{a}=2 \mathrm{~h}$ for all other temperatures. (a) Local Co (n.n) concentration (blue dots: Co K-edge, orange dots: $\mathrm{Pt}_{3}$ edge, dashdotted lines: expected values for an ideal $\mathrm{L} 1_{0}$ phase) and (b) n.n distances as a function of $T$ (same color coding as before).

measured data and fits is good. However, quantitative analysis of the measurements reveals that the CoPt alloy exhibits some rather peculiar features. First, note that the total number of next neighbors is much smaller than what is expected for a bulk fcc metal $(N=12)$, which is shown in Fig. 3(c). In addition, we also find systematic variations with concentration and edge type: the coordination number decreases almost linearly with Co concentration. At the same time, the overall number of next neighbors seems to be slightly larger at the Pt edge. Knowledge of the number of next neighbors of each type allows to calculate a local concentration and can be used to assess the degree of short range ordering. As becomes clear in Fig.3(d), neither $\mathrm{L}_{2}$ nor $\mathrm{L} 1_{0}$ phases (expected values for perfect short range order are marked with crosses for both edges) can be evidenced. Within measurement uncertainty, local Co and Pt concentrations, i.e., the number of Co neighbors surrounding either a $\mathrm{Pt}$ or Co atoms, are identical and match the global concentration, which yields strong evidence for random mixing of the constituents ${ }^{26}$. This can additionally be checked by analyzing the n.n distances. Considering the alloy to be chemically disordered, we expect $d$ to increase monotonuously with the Pt content ${ }^{26}$. This is indeed what we observe, as shown in Fig.4(e-h). Note that we find an identical behavior for Co-Pt bond lengths at the $\mathrm{Co}$ and at the $\mathrm{Pt}$ edge which illustrates the viability of our fit strategy. These experimental bond lengths resulting from our EXAFS fits can finally be compared with theoretical predictions. In the present case, we calculated the expected concentration dependent values of $d_{\mathrm{Co}-\mathrm{Co}}, d_{\mathrm{Co}-\mathrm{Pt}}=d_{\mathrm{Pt}-\mathrm{Co}}$ and $d_{\mathrm{Pt}-\mathrm{Pt}}$ for a randomly alloyed CoPt bulk system using MD simulations based on the MEAM. The results are shown in Fig. 3(e-h). While very good agreement of the differential increase $\mathrm{d} d / \mathrm{d} c(\mathrm{Pt})$ is observed, all experimental data display a constant offset $\delta$. The discrepancy found for Pt-Pt bond lengths is rather small $(\delta \simeq 0.03 \AA)$, it increases for $\mathrm{CoPt}$ bonds $(\delta \simeq 0.08 \AA)$ and becomes as large as $\delta \simeq 0.15 \AA$ for Co-Co bonds, a relative contraction of approximately $6 \%$.

\section{In-situ annealing experiments}

The absence of chemical ordering, the small coordination numbers as well as the bond-length contractions described in the previous paragraph require closer analysis. In a first step, in order to check for a possible quenching of as-deposited samples into metastable, far from equilibrium states (e.g., due to rapid cooling at the end of the growth process), we complemented our experiments with an in-situ XAS annealing study performed on sample S3, which is characterized by almost equiatomic composition $\left(\mathrm{Co}_{51} \mathrm{Pt}_{49}\right)$. As shown in Fig. 5, the data gained upon annealing up to $1000 \mathrm{~K}\left(t \simeq 2 \mathrm{~h}\right.$ and $T \simeq 600^{\circ} \mathrm{C}$ are typically employed to achieve $\mathrm{L} 1_{0}$ ordering in $\mathrm{CoPt}$ thin films), do not hint at significant changes in the lattice parameters, nor do they provide any evidence for a pronounced, systematic evolution of the system. A very small decrease of the relative amount of Co n.n. is observed at the $\mathrm{Pt}$ as well as at the Co edge and might be ascribed to partial Co oxidation. In fact, the appearance of a shallow peak at small distance in the $\chi(r)$ data supports this hypothesis. Considering the long annealing times employed, we conclude that as-deposited samples are already in a low lying energy state and that chemical ordering is indeed strongly impeded.

\section{Vibrational properties and Debye-Waller factor}

Besides giving access to changes in local order and average interatomic distances, our annealing study also provides information on the vibrational properties of the nanoalloy via calculation of the temperature dependent Debye-Waller factor $\sigma(T)$. This yields a valuable consistency check for our EXAFS analysis, as the aforementioned contraction of bond-lengths should also become manifest in a change of the vibrational signature of the system. Using the equipartition theorem, a link between the characteristic frequency of an ensemble of uncoupled, quantized harmonic oscillators with energies 
TABLE II. Einstein temperatures $\theta_{E}(\mathrm{~K})$ : bulk literature values for $\mathrm{Co}$ and $\mathrm{Pt}^{49,50}$ and calculated data for the present CoPt nanoalloy. MEAM results are given in parenthesis.

\begin{tabular}{lccc}
\hline \hline & bond type & Co edge & Pt edge \\
\hline Co bulk hcp/fcc & Co-Co & $295 / 286(265)$ & - \\
Pt bulk & Pt-Pt & & $179(173)$ \\
CoPt-SrTiO $_{3}$ & Co-Co & $405(409)$ & - \\
& Co-Pt & $396(412)$ & $416(412)$ \\
& Pt-Pt & - & $242(248)$ \\
\hline \hline
\end{tabular}

$E_{i}=n_{i}\left(\hbar \omega_{E}+1 / 2\right)$, i.e., the Einstein model, and the width of the distribution of interatomic distances $\sigma$ is readily established:

$$
\sigma^{2}(T)=\frac{\hbar}{M \omega_{E}} \operatorname{coth}\left(\beta \hbar \omega_{E} / 2\right)
$$

Here, $M$ denotes the reduced mass of the absorberscatterer pair, $\omega_{E}$, the Einstein frequency, and $\beta=$ $1 / k_{B} T$. For ease of comparison with literature values, we used a frequency to temperature conversion: $k_{B} \theta_{E}=$ $\hbar \omega_{E}$. This approach has been used extensively in EXAFS analysis and usually results in a rather good description of the experimental data, as $\sigma(T)$ is not impacted by the details of the phonon density of states ${ }^{47}$.

We adopted the textbook approach for description of $\sigma(T)$ and decomposed the latter into a dynamic and a temperature independent static part: $\sigma(T)^{2}=\sigma_{s}^{2}+$ $\sigma_{d}(T)^{2}$. In contrast to the strategy used in the last section, EXAFS data were fitted using two different $\sigma$ values. Calculated EXAFS $\sigma_{\mathrm{Co}-\mathrm{Co}}, \sigma_{\mathrm{Co}-\mathrm{Pt}}, \sigma_{\mathrm{Pt}-\mathrm{Co}}$ and $\sigma_{\mathrm{Pt}-\mathrm{Pt}}$ as well as fits to the experimental and MEAM results using eq. 1 are shown in Fig.5 (Table II provides a survey of the calculated Einstein temperatures). A comparison of our MEAM modeling with EXAFS literature data gained on bulk Co and $\mathrm{Pt}$ shows good agreement and underpins the validity of our approach. As expected, $\sigma(T)$ of the alloy differs significantly from the aforementioned elemental data. While the values show significant scattering (which results from the use of two different $\sigma$ values at each edge) the Einstein temperatures deduced from our experiments are very close to the bulk MEAM predictions (Table II). Within experimental uncertainty, the nanoalloy thus behaves as a bulk system. This finding is at odds with the pronounced bond length shortening described in a previous section of this paper, which should result in a shift of the characteristic frequency to higher values. As will be shown in the next section, a closer analysis of the static Debye-Waller factor $\sigma_{\mathrm{s}}$ might provide the key for a better understanding of the XAS data.
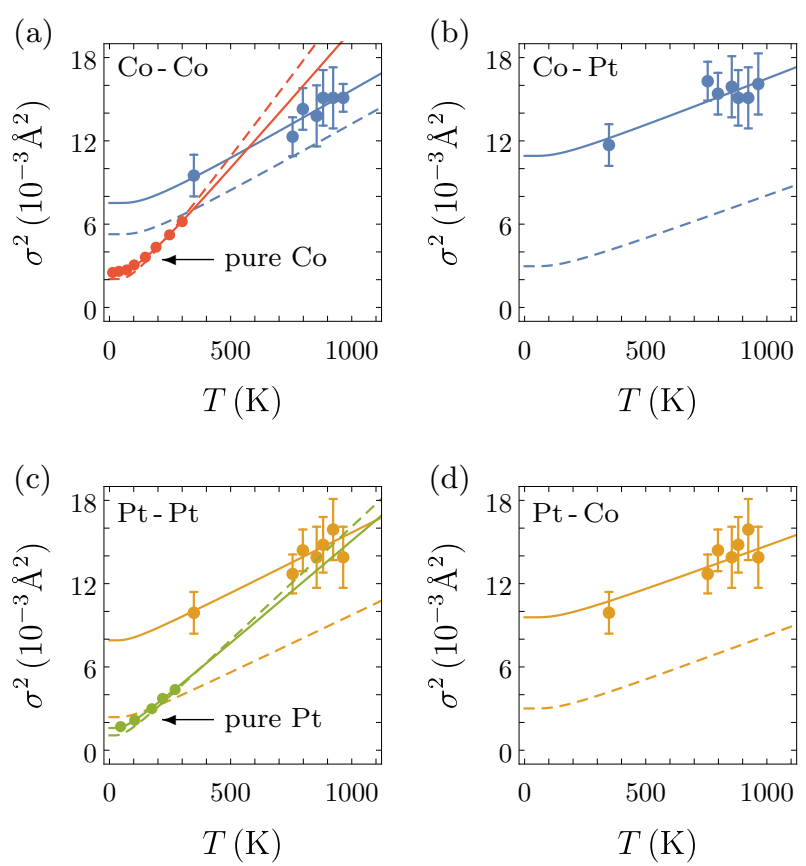

FIG. 5. Evolution of the Debye-Waller EXAFS parameter $\sigma^{2}$ with temperature. Measurements performed at the (a-b) Co $\mathrm{K}$ edge (blue) and (c-d) $\mathrm{Pt} \mathrm{L}_{3}$ edge (orange) as well as literature data gained on pure Co and $\mathrm{Pt}$ systems (red and green) and fits to eq. 1 (fits to MEAM simulations: dashed lines, fits to experimental data: solid line).

\section{Structural disorder}

As can be seen in Fig. 5, a comparison of our experimental CoPt data with MEAM-based simulations show a large constant offset, i.e., unexpectedly large $\sigma_{\mathrm{s}}$ values revealing the presence of pronounced structural disorder. In fact, detailed analysis of the XAS data confirms this observation. A subtle hint at the poor crystallinity of the CoPt phase has already been identified in the XANES, where, at the Co-edge, the double peaks B1 and B2 merge into a single maximum with increasing Co concentration ${ }^{20,51}$. Note that a similar vanishing of the double peak was reported in experiments on $\mathrm{Co}_{50} \mathrm{Pt}_{50}$ nanoparticles with sizes of approximately $2 \mathrm{~nm}^{44}$. Further qualitative confirmation for the low degree of crystallinity can be obtained by closer inspection of the Fourier transformed $\chi(r)$ spectra. As shown in Fig.3(a-b), all information beyond the first coordination shell is averaged out and the spectra become essentially flat. The effect becomes more pronounced with increasing Co concentration, it therefore seems not plausible to link it to a concentration gradient in the sample. More likely, this observation results from a severe loss of structural order, as has been reported in studies dealing with amorphous ultrathin films ${ }^{52}$, irradiated nanoparticles ${ }^{51}$ and ultrasmall embedded nanowires ${ }^{20}$. This is further supported by the small coordination numbers and shifts 
(a)

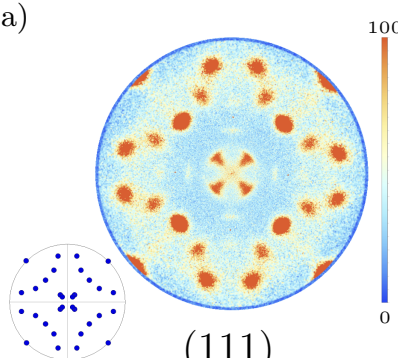

(d)

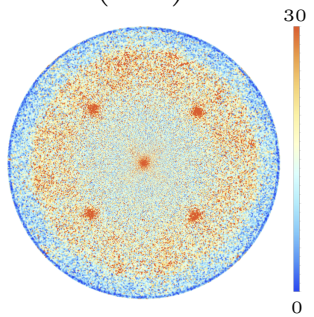

(b)

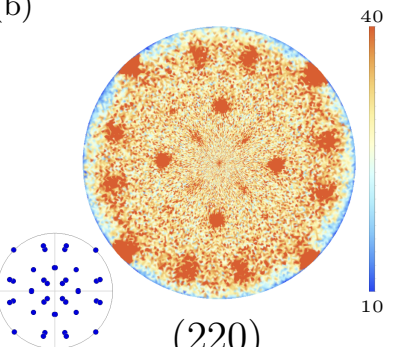

(e)

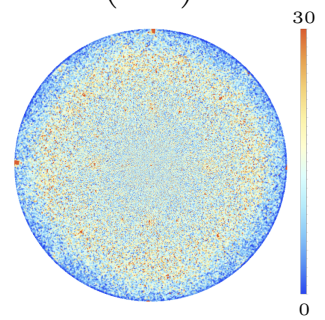

(c)

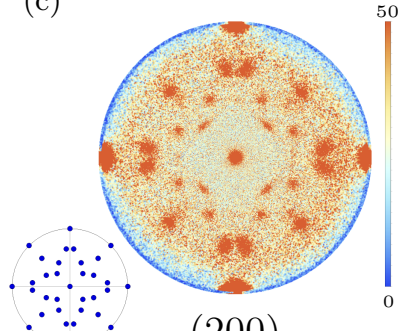

(f)

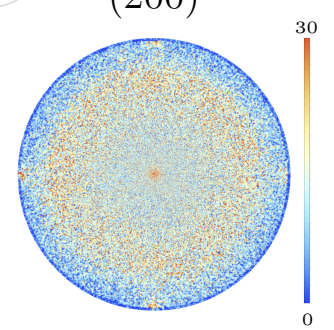

$(-1011)$

(g)

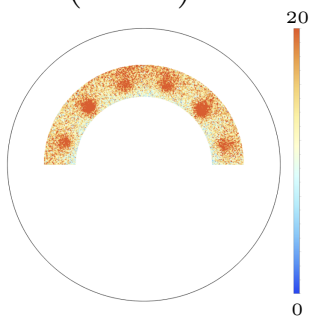

FIG. 6. Pole figure measurements. (a-c) (111), (220) and (200) poles in a Pt-rich sample (S1) as well as predictions using three different crystallite orientations (inset). (d-g) (111), (220) (200) and (-1011) poles in a Co-rich sample (S4).

in n.n. distances observed in our EXAFS analysis. At first glance, the bond length contractions might be conceived as a consequence of the small size of the bimetallic inclusions. Indeed, capillary pressures are well known to induce bondlength changes up to several percent ${ }^{53}$. However, the diameters of the rods appears too large to induce such strong shifts. In addition, the behaviour of embedded structures is expected to be different from freestanding clusters as they do not exhibit a free surface but an interfacial layer with epitaxial coupling to the matrix, going along with the creation of metallic bonds ${ }^{54}$. Furthermore, as shown in the last section, we find no evidence for bond-stiffening, which should result from a reduction of the interatomic distances. The coordination numbers shown in Fig. 3(c) are even more striking. While they are close to ten at the Pt edge for higher Pt concentrations (which appears reasonable for such small nanowires considering their high surface to volume ratio), we observe a pronounced decrease with Co concentration. Especially at the Co edge, the values become too small to be explained by finite size effects. It is clear that neither the few clusters seen in TEM nor minute amounts of Co atoms incorporated into the matrix can induce such a behavior. As already highlighted, we find no evidence for Co oxidation and we also emphasize that the data cannot be fitted by taking interdiffusion of matrix elements such as Ti into consideration. The poor crystallinity of the embedded CoPt phase can however provide an explanation for these observations. In fact, large degrees of structural disorder are known to induce a breakdown of the Gaussian approximation used for EXAFS fitting, which can result in an underestimation of n.n. distances and coordination numbers ${ }^{47}$. In the present case, the disordering appears to be quite pronounced, as the use of higher cumulants did not yield any improvement of the fits. As will be shown in the next paragraph, character- ization of the samples via XRD further underpins this hypothesis and suggests a progressive and important loss of crystallinity of the embedded phase with increasing Co content.

\section{Matrix-induced fcc $\rightarrow$ hcp transitions}

\section{X-ray diffraction (XRD) analysis}

In addition to the HR-TEM and XAS measurements presented so far, we collected XRD data, combining $\theta-2 \theta$ scans and pole figure measurements to gain additional information regarding the crystallographic properties of our samples. $\theta-2 \theta$ data confirm the homoepitaxial growth of STO on the single crystal substrate (data not shown) and corroborate our HR-TEM analysis. The scans also provide information on the embedded $\mathrm{CoPt}$ phase. A shallow, broad peak attributed to diffraction from CoPt (111) planes is observed around $2 \theta \simeq 41^{\circ}$ and shifts with concentration. The derived lattice parameters follow bulk predictions ${ }^{26}$ and we find no evidence for large OP strains that were reported in systems with pure cube-on-cube epitaxy, such as $\mathrm{Ni}$ or $\mathrm{CoNi}$ nanowires in $\mathrm{SrTiO}_{3}{ }^{20,25,55}$. In fact, in the present system, a more complex epitaxial relationship between the matrix and the nanoalloy was identified. Figure 6 shows pole figure measurements performed on a $\mathrm{Pt}$ - and a Corich nanocomposite (S1 and $\mathrm{S} 4$, respectively). As can be seen in Fig.6(a-c), the Pt-rich sample is characterized by a pronounced texturing of the metallic phase. The data can be well reproduced by using three crystallite orientations: the cube-on-cube orientation and two fourfold degenerated ones obtained by a rotation around the $[-110]_{\text {STO }}$ axis leading to $[111]_{\mathrm{CoPt}} / /[110]_{\mathrm{STO}}$ and $[11-$ $1]_{\mathrm{CoPt}} / /[111]_{\mathrm{STO}}$, respectively (i.e., obtained by a $35.26^{\circ}$ 

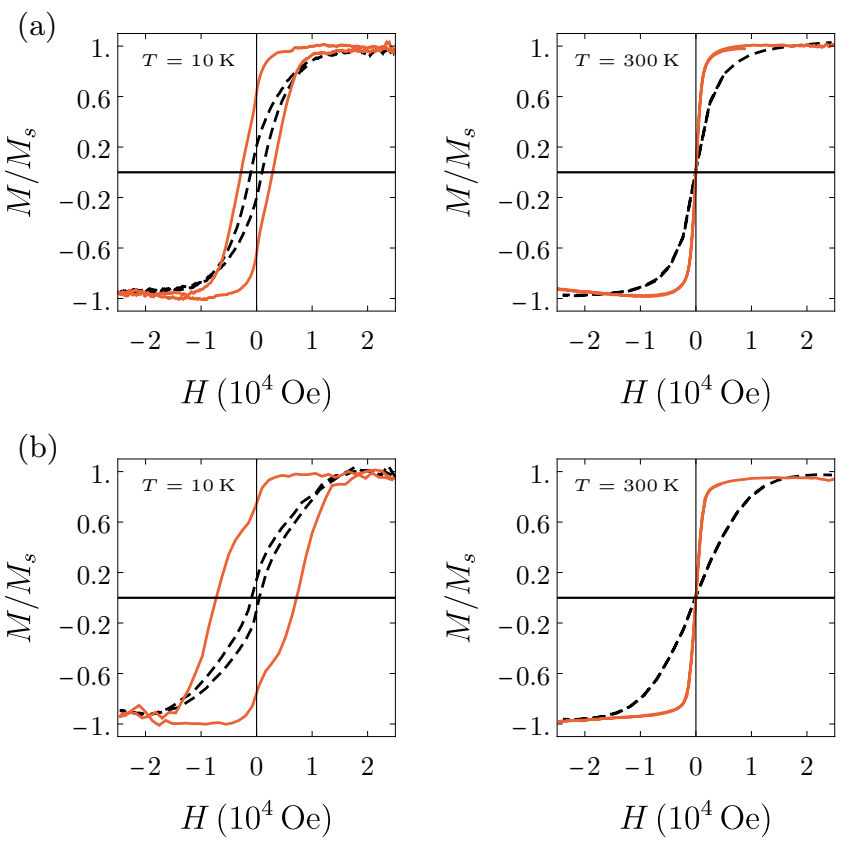

(c)
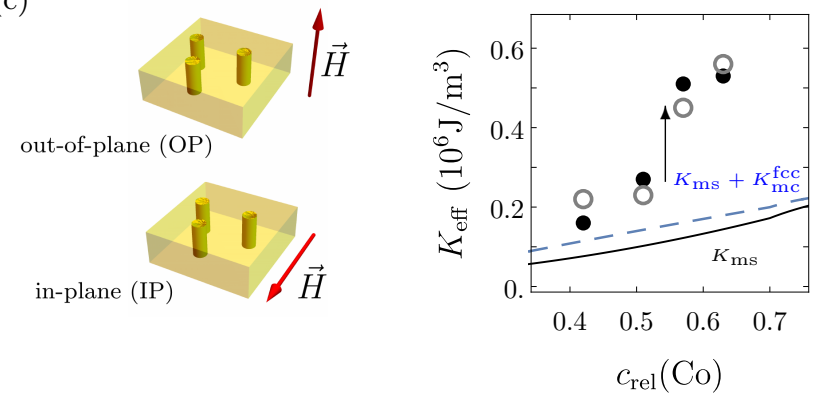

FIG. 7. Temperature dependent magnetometry measurements: (a) At $10 \mathrm{~K}$ (left) and $300 \mathrm{~K}$ (right) in IP (black, dashed line) OP geometries (red, solid line) below $c_{\text {crit }}$ (sample S3) and (b) At $10 \mathrm{~K}$ (left) and $300 \mathrm{~K}$ (right) in IP (black, dashed line) OP geometries (red, solid line) above $c_{\text {crit }}$ (sample S5). (c) IP and OP measurement geometries employed in this work (left). Effective uniaxial anisotropy $K_{\text {eff }}$ calculated from the magnetization loops at $10 \mathrm{~K}$ (gray circles) and $300 \mathrm{~K}$ (black dots). The magnetostatic anisotropy $K_{\mathrm{ms}}$ (black solid line) and magnetocrystalline $K_{\mathrm{mc}}$ fcc contributions (blue dashed curve) are shown as well (right).

and $-70.52^{\circ}$ rotation around the $[-110]_{\mathrm{STO}}$, respectively), as shown in Fig. 6.

Surprisingly, the texture changes dramatically with increasing Co content of the samples, as can be seen by comparing Fig. 6(a-c) with Fig. 6(d-f). While the intensity of the poles decreases gradually until reaching approximately $c_{\text {crit }}(\mathrm{Co}) \simeq 0.55$ (which provides additional support for a decreased degree of crystallinity) fundamental structural changes occur once the aforementioned critical Co concentration is reached. While almost all information is lost in the (220) and (200) pole figures, the (111) pole figure shows faint peaks corresponding to a cube on cube epitaxy with an additional central pole.
The latter is the signature of an unexpected hcp phase with c-axis oriented out of plane. This becomes apparent in Fig. 6(g), where hcp (-1011) peaks (Fig.6(g)) with twelve-fold symmetry can clearly be identified. Note that this observation is further corroborated by our HR-TEM data, where characteristic lattice spacings corresponding to a chemically disordered $\mathrm{CoPt}$ hcp phase were identified (data not shown).

\section{Magnetic anisotropy}

The columnar structure of the embedded CoPt phase, as well as the concentration dependent fcc $\rightarrow$ hcp texture changes evidenced in the last section, are both expected to have a marked impact on the magnetic anisotropy of the composite. Analysis of the magnetic properties of the CoPt-SrTiO 3 thin films as a function of the composition thus provides supplementary information that can be linked to the structural and morphological data presented so far. Temperature dependent magnetization curves were gathered on samples S1-S5. Exemplary results are shown in Fig. 7(a-b) (samples S3 and S5) for IP and OP configurations (Fig. 7(c)). At room temperature, all nanocomposites exhibit superparamagnetic behavior, while at $10 \mathrm{~K}$, the ferromagnetic characteristics are retrieved. Pt-rich samples exhibit coercive fields $2 \mathrm{kOe} \lesssim H_{\mathrm{c}} \lesssim 3 \mathrm{kOe}$, which increase slightly with Co concentration. Beyond $c_{\text {crit }}$, the hysteresis loops change dramatically. As shown in Fig. 7(b), the low temperature OP data hint at a much larger coercive field $H_{\mathrm{c}} \gtrsim 10 \mathrm{kOe}$. In addition, we observe a small "shoulder", which suggests that the sample is composed of two different populations of magnetic nano-objects with soft and hard magnetic properties, respectively. Qualitatively, this is in agreement with our structural analysis: at low concentrations, the samples consist of chemically disordered fcc-textured nanowires and present a moderate anisotropy, which results mainly from the high aspect ratio of the embedded structures. Above $c_{\text {crit }}$, a partial fcc $\rightarrow$ hcp transformation occurs, possibly accompanied by an amorphization of parts of the nanoalloy ${ }^{20}$. The c-axis of the hcp phase, an easy magnetic axis, is oriented in OP direction which gives rise to a pronounced anisotropy increase. To assess this quantitatively we calculated $K_{\text {eff }}=\mu_{0} \int_{0}^{H_{s}} d H\left(M_{\perp}(H)-M_{\|}(H)\right)$. The values are shown in Fig. 7(c). For small Co concentrations, a uniaxial anisotropy based solely on magnetostatic contributions $K_{m s}=\frac{\mu_{0}}{4} M_{s}^{2}(1-3 p)$ provides a reasonable description of $K_{\text {eff }}$. Deviations occur, among other things, from the difficulty to correctly determine the porosity $p$ of the sample. Note also that the fcc phase has an easy axis along the [111] direction, but is magnetically rather soft and thus, has little impact on the final value of $K_{\mathrm{eff}}{ }^{56}$. In contrast, for Co concentrations above $c_{\text {crit }}$, an increase of $K_{\text {eff }}$ by a factor of more than two is seen, which is clearly too large to be rationalized by the slow growth of $K_{\mathrm{ms}}$ with Co content. We 
ascribe this jump to a magnetocrystalline contribution to the energy of the system, that stems from the creation of a significant amount of hcp CoPt. Note that we do not see any pronounced temperature dependence of $K_{\text {eff }}$, as would be expected for pure hcp Co, where $K_{\mathrm{mc}, 1}$ is nearly halved when increasing the temperature from $10 \mathrm{~K}$ to $300 \mathrm{~K}^{57}$. Unfortunately, data describing the magnetic properties of chemically disordered CoPt alloys are scarce. Magnetocrystalline anisotropy constants of hcp CoPt have been determined by Bolzoni et al. ${ }^{58}$. For bulk CoPt, the hcp phase is found stable in a concentration range $0.8 \lesssim c(\mathrm{Co})<1$ with $0.6 \mathrm{MJ} / \mathrm{m}^{3} \lesssim$ $K_{\mathrm{mc}, 1}+2 K_{\mathrm{mc}, 2} \lesssim 1.1 \mathrm{MJ} / \mathrm{m}^{3}$. To assess these constants independently, we fitted our IP data at $300 \mathrm{~K}$ using a simple hard axis magnetization model ${ }^{59}$, considering exclusively magnetostatic and magnetocrystalline contributions: $\mu_{0} H(m) M_{s}=2\left(K_{\mathrm{mc}, 1}+K_{\mathrm{ms}}\right) m+4 K_{\mathrm{mc}, 2} m^{3}$, with $m=M / M_{s}$ and $K_{\mathrm{eff}}=K_{\mathrm{mc}, 1}+K_{\mathrm{mc}, 2}+K_{\mathrm{ms}}$. From the fit, we get $K_{\mathrm{mc}, 1}=2.3 \cdot 10^{5} \mathrm{~J} / \mathrm{m}^{3}$ while $K_{\mathrm{mc}, 2}=$ $1.5 \cdot 10^{5} \mathrm{~J} / \mathrm{m}^{3}$, and thus $K_{\mathrm{mc}, 1}+2 K_{\mathrm{mc}, 2} \simeq 0.5 \mathrm{MJ} / \mathrm{m}^{3}$. This is compatible with the data provided by Bolzoni et al.. and thus provides additional quantitative evidence for the creation of the hcp CoPt phase.

\section{CONCLUSION}

To summarize, we showed that sequential deposition of sub-monolayer amounts of Co, $\mathrm{Pt}$ and STO on $\mathrm{SrTiO}_{3}(001)$ substrates results in self-assembly of CoPt-SrTiO 3 nanocomposites, with ultrasmall, shape anisotropic bimetallic CoPt nanoalloys, vertically embedded in $\mathrm{SrTiO}_{3}$. Our XAS analysis confirms the metallic nature of the CoPt phase and provides strong evidence for the absence of oxide or compound formation. However, in contrast to other well established growth strategies, such as low energy cluster deposition for example, the present synthesis approach gives rise to a metallic CoPt nanoalloy phase presenting several unusual prop- erties. Its two most striking features are the complete absence of chemical ordering, even after additional annealing steps, and the pronounced degree of structural disorder, which is linked to strong nanocrystalline texturing. While at low Co concentrations, several different fcc orientations coexist, higher Co concentrations give rise to the additional creation of an hcp phase, which is confirmed by magnetometry measurements. Although past work has already emphasized the impact of size on the order-disorder phase transition in $\mathrm{CoPt}^{29}$, our present study highlights the outstanding role played by the matrix into which the alloy is embedded, even in absence of oxidation and compound formation. It appears that, in contrast to earlier results obtained on CoPt nanoalloys embedded in amorphous matrices, interactions and constraints imposed by a single crystal via vertical epitaxy can strongly affect the thermodynamic and structural properties of ultrasmall CoPt alloys. To what extent the present experiments can be generalized to other types of nanoalloys displaying ordered phases, such as $\mathrm{CuAu}$ or FePt for example, remains to be fully elucidated.

\section{ACKNOWLEDGMENTS}

We acknowledge SOLEIL for provision of synchrotron beamtime (proposal ID: 20171296). The authors thank the IMPMC, CNRS-Sorbonne Université, for access to the TEM facilities and Dr. J.-M. Guigner for his help. We thank S. Chenot for his help and UHV expertise and the staff of the MPBT platform of Sorbonne Université for their support. The STEM studies were carried out on a microscope acquired as part of the TEMPOS project (ANR-10-EQPX-0050). This work was supported by French state funds managed by the ANR Investissements d'Avenir (ANR-11-IDEX-0004-02) within the framework of the Cluster of Excellence MATISSE. M. Hennes acknowledges financial support from the French Embassy in Berlin and CampusFrance. We thank E. Jal for a critical review of the manuscript.
* marcel.hennes@sorbonne-universite.fr

$\dagger$ franck.vidal@sorbonne-universite.fr

¥ Currently at: Sorbonne Université, CNRS, Laboratoire de Chimie Physique-Matière et Rayonnement (LCPMR), F75005 Paris, France

1 A. Meldrum, Jr. Haglund, R. F., L. A. Boatner, and C. W. White. Nanocomposite materials formed by ion implantation. Advanced Materials, 13(19):1431-1444, 2001.

2 M. Gaudry, J. Lermé, E. Cottancin, M. Pellarin, J. L. Vialle, M. Broyer, B. Prével, M. Treilleux, and P. Mélinon. Optical properties of $\left(\mathrm{Au}_{x} \mathrm{Ag}_{x-1}\right)_{n}$ clusters embedded in alumina: Evolution with size and stoichiometry. Phys. Rev. B, 64:085407, 2001.

${ }^{3}$ G. Celep, E. Cottancin, J. Lermé, M. Pellarin, L. Arnaud, J. R. Huntzinger, J. L. Vialle, M. Broyer, B. Palpant, O. Boisron, and P. Mélinon. Optical properties of cop- per clusters embedded in alumina: An experimental and theoretical study of size dependence. Phys. Rev. B, 70: 165409, 2004.

4 Venkata Sai Kiran Chakravadhanula, Yogendra Kumar Mishra, Venkata Girish Kotnur, Devesh Kumar Avasthi, Thomas Strunskus, Vladimir Zaporotchenko, Dietmar Fink, Lorenz Kienle, and Franz Faupel. Microstructural and plasmonic modifications in $\mathrm{AgTiO}_{2}$ and $\mathrm{AuTiO}_{2}$ nanocomposites through ion beam irradiation. Beilstein Journal of Nanotechnology, 5:1419-1431, 2014.

5 Xiaoyuan $\mathrm{Hu}$, David G. Cahill, and Robert S. Averback. Burrowing of $\mathrm{Pt}$ nanoparticles into $\mathrm{SiO}_{2}$ during ion-beam irradiation. Journal of Applied Physics, 92(7):3995-4000, 2002.

6 A. Klimmer, P. Ziemann, J. Biskupek, U. Kaiser, and M. Flesch. Size-dependent effect of ion bombardment on 
$\mathrm{Au}$ nanoparticles on top of various substrates: Thermodynamically dominated capillary forces versus sputtering. Phys. Rev. B, 79:155427, 2009.

7 Udai B. Singh, D. C. Agarwal, S. A. Khan, S. Mohapatra, H. Amekura, D. P. Datta, Ajay Kumar, R. K. Choudhury, T. K. Chan, Thomas Osipowicz, and D. K. Avasthi. Synthesis of embedded $\mathrm{Au}$ nanostructures by ion irradiation: influence of ion induced viscous flow and sputtering. Beilstein J Nanotechnol, 5:105-110, 2014.

8 M. Hennes, A. M. Jakob, F. Lehnert, U. Ross, A. Lotnyk, and S. G. Mayr. Nanometer-resolved quantification of mechanical response in nanoparticle-based composites. Nanoscale, 8:9398-9404, 2016.

9 Marisa Mäder, Thomas Höche, Jürgen W. Gerlach, Susanne Perlt, Jens Dorfmüller, Michael Saliba, Ralf Vogelgesang, Klaus Kern, and Bernd Rauschenbach. Plasmonic activity of large-area gold nanodot arrays on arbitrary substrates. Nano Letters, 10(1):47-51, 2010.

10 D J Sellmyer, M Zheng, and R Skomski. Magnetism of Fe, Co and Ni nanowires in self-assembled arrays. Journal of Physics: Condensed Matter, 13(25):R433-R460, 2001.

11 Jie Yao, Zhaowei Liu, Yongmin Liu, Yuan Wang, Cheng Sun, Guy Bartal, Angelica M. Stacy, and Xiang Zhang. Optical negative refraction in bulk metamaterials of nanowires. Science, 321(5891):930-930.

12 M. Hennes, V. Schuler, X. Weng, J. Buchwald, D. Demaille, Y. Zheng, and F. Vidal. Growth of vertically aligned nanowires in metal-oxide nanocomposites: kinetic monte-carlo modeling versus experiments. Nanoscale, 10: 7666-7675, 2018.

13 L. Mohaddes-Ardabili, H. Zheng, S. B. Ogale, B. Hannoyer, W. Tian, J. Wang, S. E. Lofland, S. R. Shinde, T. Zhao, Y. Jia, L. Salamanca-Riba, D. G. Schlom, M. Wuttig, and R. Ramesh. Self-assembled single-crystal ferromagnetic iron nanowires formed by decomposition. 3: $533 \mathrm{EP}-, 2004$.

14 F. Vidal, Y. Zheng, J. Milano, D. Demaille, P. Schio, E. Fonda, and B. Vodungbo. Nanowires formation and the origin of ferromagnetism in a diluted magnetic oxide. Applied Physics Letters, 95(15):152510, 2009.

15 F. Vidal, Y. Zheng, P. Schio, F. J. Bonilla, M. Barturen, J. Milano, D. Demaille, E. Fonda, A. J. A. de Oliveira, and V. H. Etgens. Mechanism of localization of the magnetization reversal in $3 \mathrm{~nm}$ wide Co nanowires. Phys. Rev. Lett., 109:117205, 2012.

16 P Schio, F J Bonilla, Y Zheng, D Demaille, J Milano, A J A de Oliveira, and F Vidal. Grain structure and magnetic relaxation of self-assembled Co nanowires. Journal of Physics: Condensed Matter, 25(5):056002, 2013.

17 Jijie Huang, Leigang Li, Ping Lu, Zhimin Qi, Xing Sun, Xinghang Zhang, and Haiyan Wang. Self-assembled $\mathrm{CoBaZrO}_{3}$ nanocomposite thin films with ultra-fine vertically aligned Co nanopillars. Nanoscale, 9:7970-7976, 2017.

18 Vivien Schuler, Francisco Javier Bonilla, Dominique Demaille, Alessandro Coati, Alina Vlad, Yves Garreau, Michèle Sauvage-Simkin, Anastasiia Novikova, Emiliano Fonda, Sarah Hidki, Victor Etgens, Franck Vidal, and Yunlin Zheng. Huge metastable axial strain in ultrathin heteroepitaxial vertically aligned nanowires. Nano Research, 8(6):1964-1974, 2015.

19 Qing Su, Wenrui Zhang, Ping Lu, Shumin Fang, Fauzia Khatkhatay, Jie Jian, Leigang Li, Fanglin Chen, Xinghang Zhang, Judith L. MacManus-Driscoll, Aiping Chen,
Quanxi Jia, and Haiyan Wang. Self-assembled magnetic metallic nanopillars in ceramic matrix with anisotropic magnetic and electrical transport properties. ACS Applied Materials \& Interfaces, 8(31):20283-20291, 2016.

${ }^{20}$ X. Weng, M. Hennes, A. Coati, A. Vlad, Y. Garreau, M. Sauvage-Simkin, E. Fonda, G. Patriarche, D. Demaille, F. Vidal, and Y. Zheng. Ultrathin Ni nanowires embedded in $\mathrm{SrTiO}_{3}$ : Vertical epitaxy, strain relaxation mechanisms, and solid-state amorphization. Phys. Rev. Materials, 2: $106003,2018$.

21 Leigang Li, Liuyang Sun, Juan Sebastian Gomez-Diaz, Nicki L. Hogan, Ping Lu, Fauzia Khatkhatay, Wenrui Zhang, Jie Jian, Jijie Huang, Qing Su, Meng Fan, Clement Jacob, Jin Li, Xinghang Zhang, Quanxi Jia, Matthew Sheldon, Andrea Alu, Xiaoqin Li, and Haiyan Wang. Selfassembled epitaxial Au-oxide vertically aligned nanocomposites for nanoscale metamaterials. Nano Letters, 16(6): 3936-3943, 2016.

22 Seiji Kawasaki, Ryota Takahashi, Takahisa Yamamoto, Masaki Kobayashi, Hiroshi Kumigashira, Jun Yoshinobu, Fumio Komori, Akihiko Kudo, and Mikk Lippmaa. Photoelectrochemical water splitting enhanced by self-assembled metal nanopillars embedded in an oxide semiconductor photoelectrode. Nature Communications, 7:11818 EP -, 2016.

23 Mikk Lippmaa, Seiji Kawasaki, Ryota Takahashi, and Takahisa Yamamoto. Noble metal clustering and nanopillar formation in an oxide matrix. Japanese Journal of Applied Physics, 59(1):010501, 2019.

24 Francisco Javier Bonilla, Anastasiia Novikova, Franck Vidal, Yunlin Zheng, Emiliano Fonda, Dominique Demaille, Vivien Schuler, Alessandro Coati, Alina Vlad, Yves Garreau, Michèle Sauvage Simkin, Yves Dumont, Sarah Hidki, and Victor Etgens. Combinatorial growth and anisotropy control of self-assembled epitaxial ultrathin alloy nanowires. ACS Nano, 7(5):4022-4029, 2013.

25 V Schuler, J Milano, A Coati, A Vlad, M Sauvage-Simkin, Y Garreau, D Demaille, S Hidki, A Novikova, E Fonda, $\mathrm{Y}$ Zheng, and F Vidal. Growth and magnetic properties of vertically aligned epitaxial CoNi nanowires in $(\mathrm{Sr}, \mathrm{Ba}) \mathrm{TiO}_{3}$ with diameters in the 1.8-6 nm range. Nanotechnology, 27 (49):495601, 2016.

26 Pascal Andreazza, Véronique Pierron-Bohnes, Florent Tournus, Caroline Andreazza-Vignolle, and Véronique Dupuis. Structure and order in cobalt/platinum-type nanoalloys: from thin films to supported clusters. Surface Science Reports, 70(2):188 - 258, 2015.

27 K. Barmak, R. A. Ristau, K. R. Coffey, M. A. Parker, and J. K. Howard. Grain growth and ordering kinetics in CoPt thin films. Journal of Applied Physics, 79(8):5330-5332, 1996.

28 R. A. Ristau, K. Barmak, L. H. Lewis, K. R. Coffey, and J. K. Howard. On the relationship of high coercivity and L10 ordered phase in CoPt and FePt thin films. Journal of Applied Physics, 86(8):4527-4533, 1999.

29 D. Alloyeau, C. Ricolleau, C. Mottet, T. Oikawa, C. Langlois, Y. Le Bouar, N. Braidy, and A. Loiseau. Size and shape effects on the order-disorder phase transition in copt nanoparticles. Nature Materials, 8:940 EP -, 2009.

${ }^{30}$ L. Favre, V. Dupuis, E. Bernstein, P. Mélinon, A. Pérez, S. Stanescu, T. Epicier, J.-P. Simon, D. Babonneau, J.M. Tonnerre, and J.-L. Hodeau. Structural and magnetic properties of CoPt mixed clusters. Phys. Rev. B, 74:014439, 2006. 
31 A. Ouerghi, J. Penuelas, C. Andreazza-Vignolle, P. Andreazza, N. Bouet, and H. Estrade-Szwarckopf. Chemical and structural aspects of CoPt silicide nanostructures grown on $\mathrm{Si}(100)$. Journal of Applied Physics, 100(12): 124310, 2006.

${ }^{32}$ R. K. Rakshit, S. K. Bose, R. Sharma, and R. C. Budhani. Giant coercivity nanodots and fractals in CoPt films grown on (001) $\mathrm{SrTiO}_{3}$ using pulsed laser deposition. Applied Physics Letters, 89(20):202511, 2006.

33 D. Suzuki, M. Ohtake, F. Kirino, and M. Futamoto. Preparation of CoPt-alloy thin films with perpendicular magnetic anisotropy on $\mathrm{MgO}(111), \mathrm{SrTiO}_{3}(111)$, and $\mathrm{Al}_{2} \mathrm{O}_{3}(0001)$ single-crystal substrates. IEEE Transactions on Magnetics, 48(11):3195-3198, 2012.

34 Akira Itabashi, Mitsuru Ohtake, Shouhei Ouchi, Fumiyoshi Kirino, and Masaaki Futamoto. Structure characterization of $\mathrm{FePd}$, FePt, and CoPt alloy thin films epitaxially grown on $\mathrm{SrTiO}_{3}(001)$ single-crystal substrates. Journal of the Magnetics Society of Japan, advpub, 2013.

35 S. Plimpton. Fast parallel algorithms for short-range molecular dynamics. J. Comp. Phys., 1995.

36 Byeong-Joo Lee and M. I. Baskes. Second nearest-neighbor modified embedded-atom-method potential. Phys. Rev. B, 62:8564-8567, 2000.

37 Jin-Soo Kim, Donghyuk Seol, Joonho Ji, Hyo-Sun Jang, Yongmin Kim, and Byeong-Joo Lee. Second nearestneighbor modified embedded-atom method interatomic potentials for the Pt-M ( $\mathrm{M}=\mathrm{Al}, \mathrm{Co}, \mathrm{Cu}, \mathrm{Mo}, \mathrm{Ni}, \mathrm{Ti}, \mathrm{V})$ binary systems. Calphad, 59:131-141, 2017.

${ }^{38}$ W. Shinoda, M. Shiga, and M. Mikami. Rapid estimation of elastic constants by molecular dynamics simulation under constant stress. Phys. Rev. B, 69:134103, 2004.

39 M. E. Tuckerman, J. Alejandre, R. López-Rendón, A. L. Jochim, and G. J. Martyna. A liouville-operator derived measure-preserving integrator for molecular dynamics simulations in the isothermalisobaric ensemble. J. Phys. A.Math. Gen., 39(19):5629.

40 E. K. Hlil, R. Baudoing-Savois, B. Moraweck, and A. J. Renouprez. X-ray absorption edges in platinum-based alloys. 2. influence of ordering and of the nature of the second metal. The Journal of Physical Chemistry, 100(8), 1996.

41 Y. S. Lee, J. Y. Rhee, C. N. Whang, and Y. P. Lee. Electronic structure of Co-Pt alloys: X-ray spectroscopy and density-functional calculations. Phys. Rev. B, 68:235111, 2003.

${ }^{42}$ M. Kuhn, T.K. Sham, J.M. Chen, and K.H. Tan. Electronic structure of $\mathrm{Cu}_{3} \mathrm{Au}$ from the $\mathrm{Cu}$ viewpoint. Solid State Communications, 75(11):861 - 865, 1990.

43 B. Moraweck, A. J. Renouprez, E. K. Hlil, and R. Baudoing-Savois. Alloying effects on x-ray absorption edges in nickel-platinum single crystals. The Journal of Physical Chemistry, 97(17):4288-4292, 1993.

44 N. Blanc, L. E. Díaz-Sánchez, A. Y. Ramos, F. Tournus, H. C. N. Tolentino, M. De Santis, O. Proux, A. Tamion, J. Tuaillon-Combes, L. Bardotti, O. Boisron, G. M. Pastor, and V. Dupuis. Element-specific quantitative determination of the local atomic order in CoPt alloy nanoparticles: Experiment and theory. Phys. Rev. B, 87:155412, 2013.
45 M. Brown, R. E. Peierls, and E. A. Stern. White lines in x-ray absorption. Phys. Rev. B, 15:738-744, 1977.

${ }^{46}$ N M Souza-Neto, A Y Ramos, H C N Tolentino, and Y Joly. Depth dependent local structures in CoPt thin films. Journal of Physics: Conference Series, 190(1): 012112, 2009.

47 Grant Bunker. Introduction to XAFS. Cambridge University Press, Cambridge, 2010.

48 B. Ravel and M. Newville. ATHENA, ARTEMIS, HEPHAESTUS: data analysis for X-ray absorption spectroscopy using IFEFFIT. Journal of Synchrotron Radiation, 12(4):537-541, 2005.

49 R Giulian, L L Araujo, P Kluth, D J Sprouster, C S Schnohr, G J Foran, and M C Ridgway. Temperaturedependent EXAFS analysis of embedded Pt nanocrystals. J Phys,.: Condens. Matter, 21:155302, 2009.

50 David J. Sprouster and Mark C. Ridgway. Ion beam formation and modification of cobalt nanoparticles. Applied Sciences, 2(2):396-442, 2012.

51 D. J. Sprouster, R. Giulian, L. L. Araujo, P. Kluth, B. Johannessen, N. Kirby, K. Nordlund, and M. C. Ridgway. Ion-irradiation-induced amorphization of cobalt nanoparticles. Phys. Rev. B, 81:155414, 2010.

52 H. Magnan, D. Chandesris, G. Rossi, G. Jezequel, K. Hricovini, and J. Lecante. Determination of the local order in amorphous cobalt films. Phys. Rev. B, 40:9989-9992, 1989.

53 G. Apai, J. F. Hamilton, J. Stohr, and A. Thompson. Extended $\mathrm{x}$-ray-absorption fine structure of small $\mathrm{Cu}$ and Ni clusters: Binding-energy and bond-length changes with cluster size. Phys. Rev. Lett., 43:165-169, 1979.

54 Guillaume Radtke, Marcel Hennes, Matthieu Bugnet, Quentin M. Ramasse, Xiaorong Weng, Dominique Demaille, Benoit Gobaut, Philippe Ohresser, Edwige Otero, Fadi Choueikani, Amlie Juhin, Philippe Sainctavit, Yunlin Zheng, and Franck Vidal. Atomic-scale study of metal-oxide interfaces and magnetoelastic coupling in selfassembled epitaxial vertically aligned magnetic nanocomposites. Advanced Materials Interfaces, 0(0):1900549.

55 M. Hennes, X. Weng, E. Fonda, B. Gallas, G. Patriarche, D. Demaille, Y. Zheng, and F. Vidal. Phase separation and surface segregation in $\mathrm{Co}-\mathrm{Au}-\mathrm{SrTiO}_{3}$ thin films: Selfassembly of bilayered epitaxial nanocolumnar composites. Phys. Rev. Materials, 3:035002, 2019.

56 S. S. A. Razee, J. B. Staunton, and F. J. Pinski. Firstprinciples theory of magnetocrystalline anisotropy of disordered alloys: Application to cobalt platinum. Phys. Rev. $B, 56: 8082-8090,1997$.

57 Fumihisa Ono. Magnetic field dependence of the magnetocrystalline anisotropy energy in hcp Co. Journal of the Physical Society of Japan, 50(8):2564-2572, 1981.

58 F. Bolzoni, F. Leccabue, R. Panizzieri, and L. Pareti. Magnetocrystalline anisotropy and phase transformation in Co-Pt alloy. IEEE Transactions on Magnetics, 20(5): 1625-1627, 1984.

59 Robert C. O'Handley. Modern magnetic materials :. Wiley,, New York :, c2000. 